\title{
Computed tomography measurements of parapneumonic effusion indicative of
} thoracentesis

\author{
B.K. Moffett*,\#, T.S. Panchabhai ${ }^{\star, \#}$, E. Anaya ${ }^{\#, \oplus, ~ R . ~ N a k a m a t s u ~}{ }^{\#,+}$, F.W. Arnold ${ }^{\#,+}$, \\ P. Peyrani ${ }^{\#,+}$, T. Wiemken ${ }^{+}$, J. Guardiola ${ }^{\#, \uparrow}$ and J.A. Ramirez ${ }^{\#,+}$
}

ABSTRACT: Patients with parapneumonic effusions (PPE) measuring $<1 \mathrm{~cm}$ by lateral decubitus radiograph (LDR) or $<5 \mathrm{~cm}$ by lateral erect radiograph (LER) do not require thoracentesis. No such data exist for chest computed tomography (CCT). The objective of this study was to identify a PPE measurement by CCT that indicates the need for thoracentesis.

A secondary data analysis of two pneumonia databases was conducted to identify patients with PPE. Measurements of PPE using LDR, LER and CCT were correlated by linear regression analysis. The clinical outcome of community-acquired pneumonia patients managed with the newly defined CCT measurement was evaluated.

PPE was identified in 419 out of 1,460 patients with possible pneumonia. PPE measurements of $1 \mathrm{~cm}$ and $5 \mathrm{~cm}$ by LDR and LER, respectively, correlated with a measurement of $2.5 \mathrm{~cm}$ by CCT. Out of 95 patients with CCT measurements $<2.5 \mathrm{~cm}, 31$ poor clinical outcomes were reported: outcome was PPE related $(n=1)$; outcome was PPE unrelated $(n=26)$; and outcome was not evaluable $(n=4)$. The single case of poor outcome also measured $<1 \mathrm{~cm}$ by LDR.

This study indicates that patients with community-acquired pneumonia and a PPE measuring $<2.5 \mathrm{~cm}$ by CCT can be managed without the need for thoracentesis.

KEYWORDS: Community-acquired pneumonia, computed tomography, outcome, pleural effusion, thoracentesis

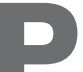

arapneumonic effusions (PPE) result from inflammation of the visceral pleura as a consequence of infection of the lung parenchyma by bacterial or viral pathogens [1-4]. These effusions occur in up to $45 \%$ of patients with community-acquired pneumonia (CAP) and have a $10 \%$ chance of progressing to an empyema with associated increased morbidity and mortality [2, 4-6]. An important step in the management of PPE is to ascertain which effusions require further diagnostic evaluation. Aspiration is recommended in patients with a PPE $>1 \mathrm{~cm}$ measured by lateral decubitus radiograph (LDR) [2, 7], or in patients with a PPE $>5 \mathrm{~cm}$ measured by lateral erect radiograph (LER) [8].

Since the 1980s, there has been a nearly 20 -fold increase in the use of computed tomography [9]. In our clinical experience, there has been an increased use of computed tomography pulmonary angiograms by emergency room physicians for evaluating patients with hypoxaemia, many of whom have CAP. Since chest computed tomography (CCT) cannot adequately discern which effusions require thoracentesis by radiographic criteria alone, and because no measurement similar to LDR has been established, these patients require subsequent imaging with LDR or LER. This is concerning as a previous study of PPE described a significant delay in time to thoracentesis while awaiting LDR [9], and a delay of successful drainage may result in increased length of stay, cost, complications and mortality $[3,4,10,11]$.

We hypothesise that since CCT demonstrates layering effects similar to LDR, it would provide an attractive early alternative in patients who have already received a CCT. Since previous studies have established a method of correlating measurements between LDR and ultrasound [12], and between LDR and LER [8], it should be possible to define a correlation between measurements with

Earn CME accreditation by answering questions about this article. You will find these at the back of the printed copy of this issue or online at www.erj.ersjournals.com/misc/cmeinfo.xhtml

\section{AFFILIATIONS}

*Depts of Medicine, Division of General Internal Medicine, Palliative Care and Medical Education, "Division of Pulmonary Critical Care and Sleep Disorders Medicine, School of Medicine,

+Division of Infectious Diseases, School of Medicine, University of Louisville, and

\#Robley Rex Veterans Administration Medical Center, Louisville, KY, USA.

\section{CORRESPONDENCE}

B.K. Moffett

Dept of Medicine

Division of General Internal Medicine Palliative Care and Medical Education School of Medicine

University of Louisville

800 Zorn Ave

Louisville

KY 40206

USA

E-mail: bryan.moffett@va.gov

Received:

Jan 112011

Accepted after revision:

April 282011

First published online:

May 262011 
LDR and CCT. This measurement can be used to define the need for thoracentesis once a PPE is documented by CCT. Therefore, we designed a study, first, to determine whether a correlation exists between PPE measurements by LDR and CCT, and LER and CCT, and secondly to evaluate whether the newly defined PPE measurements by CCT can be safely used to define the need for thoracentesis.

\section{MATERIALS AND METHODS Study design}

This was a secondary data analysis of two retrospective cohort pneumonia databases comprised of patients hospitalised from December 2005 to March 2009 at the Robley Rex Veterans Administration Medical Center (VAMC; Louisville, KY, USA). One database contained patients with the diagnosis of CAP enrolled in the Community-Acquired Pneumonia Organization (CAPO) international cohort study. The other database contained patients with diagnosis of hospital-acquired pneumonia (HAP) and ventilator-associated pneumonia (VAP) enrolled in the Hospital-Acquired Pneumonia Organization (HAPO) study. Only CAPO and HAPO patients enrolled from the VAMC were reviewed due to limitations in LDR, LER or CCT availability of the larger international database. Decisions regarding medications, laboratory testing, radiography and thoracentesis were left to the emergency room and hospital provider's discretion, and neither CAPO nor HAPO enrolment required any additional interventions. The VAMC Institutional Review Board approved both studies (CAPO 350.01 and IMPACT-HAP 519.06).

\section{Study definitions}

Pneumonia was defined as new radiographic evidence of a pulmonary infiltrate, as well as one of the following: new or increased cough with or without sputum production; temperature $<35.6^{\circ} \mathrm{C}$ or $>37.8^{\circ} \mathrm{C}$; or leukocytosis $\left(>11,000\right.$ cells $\left.\cdot \mathrm{mm}^{-3}\right)$, leukopenia $\left(<4,000\right.$ cells $\left.\cdot \mathrm{mm}^{-3}\right)$ or bandaemia $>5 \%$ [13]. Patients were defined as having CAP if their infiltrate was present on radiography within $48 \mathrm{~h}$ of admission.

\section{PPE evaluation}

Radiographs were excluded from measurements if they showed any of the following characteristics: uninterpretable due to under penetration; inability to visualise the inferior margin of the radiograph; radiographs of the incorrect side (LDR); error in type of image obtained; loculated effusion; hydropneumothorax; bilateral effusions (LER); diaphragmatic elevation (LER); effusion volume too large to determine the fluid level; or patients in the lateral position (CCT). For the purpose of comparing effusions, radiographs were excluded from analysis if they were obtained $>72 \mathrm{~h}$ apart.

All effusions were measured (in $\mathrm{cm}$ ) using a ruler incorporated into the imaging software (CPRS VistA Imaging system; VAMC) in the following manner. 1) LDR was obtained by measuring from the delineation of the fluid lamella from the air-filled lung to a point parallel to the parietal pleura at the inner reflection of the rib along a path perpendicular to the inferior margin of the radiograph (fig. 1a). 2) LER was obtained by measuring from the most inferior visible point of the contralateral sulcus to the fluid lamella using a path perpendicular to the inferior margin of the radiograph (fig. 1b). 3) CCT was obtained by measuring from the parietal pleura-fluid junction to the visceral pleurafluid junction in the middle third of the lung (from $1.5 \mathrm{~cm}$ above to $7.5 \mathrm{~cm}$ below the carina) along the mid-scapular line, where the fluid appeared thickest (fig. 1c).

\section{Patient characteristics}

For each CAP patient, the following characteristics were evaluated: demographics; radiographic findings; date and results of all thoracenteses, including $\mathrm{pH}$, total protein of serum and fluid, lactate dehydrogenase (LDH) of serum and fluid, albumin, glucose, Gram-stain and culture, and cytology.

\section{Study outcomes}

The clinical outcome of CAP patients managed with clinical observation without thoracentesis according to the newly defined CCT measurement was evaluated. Clinical failure was defined as: death within 30 days of admission; readmission within 30 days of discharge; tube thoracostomy, thoracotomy or decortication required during hospitalisation or within 30 days; or pleural fluid results consistent with complicated PPE or empyema as defined by the American College of Chest Physician guidelines [14, 15]. Data on patients with clinical failure were reviewed and discussed amongst a panel of six infectious and pulmonary disease physicians to classify the outcome as PPE related, PPE unrelated or not evaluable.
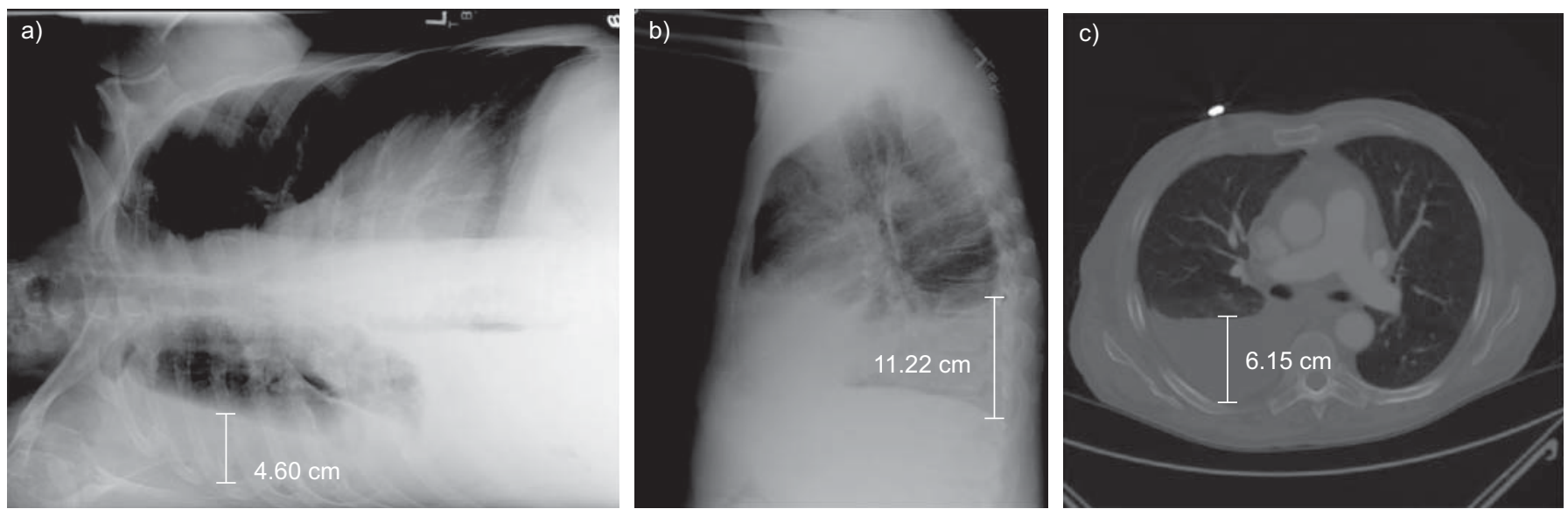

FIGURE 1. Techniques for measuring effusions using a) lateral decubitus radiograph, b) lateral erect radiograph and c) chest computed tomography. 


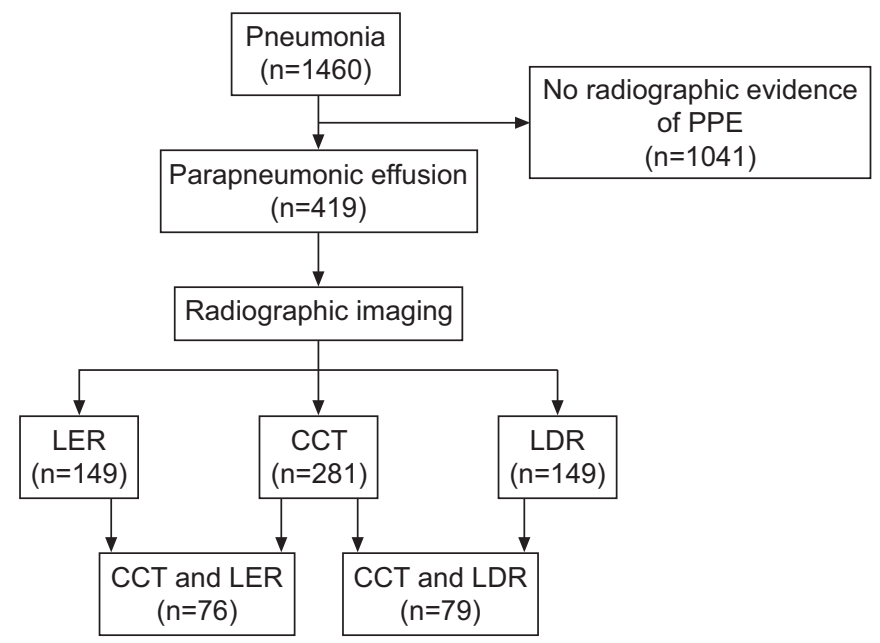

FIGURE 2. Presence of effusion in 1,460 patients with possible pneumonia and available radiography for comparison. PPE: parapneumonic effusion; LER: lateral erect radiograph; CCT: chest computed tomography; LDR: lateral decubitus radiograph.

\section{Statistical analysis}

LER, LDR and CCT measurements were compared using PASW software (version 18; SPSS Inc., Chicago, IL, USA) to generate a linear regression analysis with best-fit line and $r^{2}$ analysis. The LDR and LER measurements were defined as independent variables and the CCT measurements as dependent variables. The intersection point of the best-fit line with the defined values of the independent variables ( $1 \mathrm{~cm}$ for LDR and $5 \mathrm{~cm}$ for LER) was then evaluated for CCT and the two results were compared. The newly defined CCT value was then applied to patients enrolled in the CAPO database to define the clinical outcomes of patients with fluid collections $<2.5 \mathrm{~cm}$ by CCT.

\section{RESULTS}

\section{Correlation of PPE measurements between LDR, LER and CCT}

From a total of 1,460 patients with possible pneumonia, PPE was identified in 419 patients. The mean \pm SD age of these patients was $70.1 \pm 11.7$ yrs and $1.2 \%$ of patients were female. Of the 419 cases of PPE, $281(67.1 \%)$ were evaluated by CCT. The available

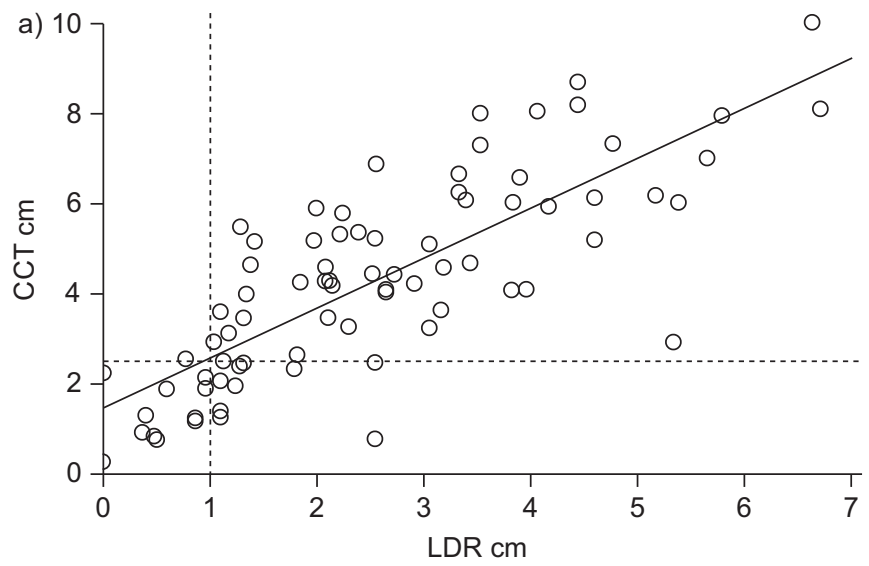

imaging modalities in patients with PPE are summarised in figure 2. In patients with PPE, a total of 79 pairs of radiographs were available for comparison of LDR and CCT, and 76 pairs of radiographs were available for comparison of LER and CCT. LDR values of $1 \mathrm{~cm}$ and LER values of $5 \mathrm{~cm}$ were correlated with CCT values of $2.5 \mathrm{~cm}$ (fig. 3).

\section{Clinical application of CCT measurements in patients with CAP and PPE}

Among the 582 patients with CAP, 240 (41.2\%) had PPE. The results of the evaluation of the 582 patients with CAP for available radiographs are presented in figure 4 . This analysis showed that 302 (51.9\%) CAP patients underwent CCT during their hospitalisation, with 245 (42.1\%) CAP patients receiving CCT within the first $48 \mathrm{~h}$ of hospitalisation. Table 1 shows the available radiographs for evaluating PPE in the 240 patients with PPE, as well as the limitations of each modality. CCT was the method used to evaluate PPE in $75.4 \%$ of cases (181 out of 240 ), while only $23.8 \%$ (57 out of 240 ) of patients with PPE were evaluated by LDR and only $37.5 \%$ (90 of 240) were evaluated by LER. After applying our new criteria of $2.5 \mathrm{~cm}$ to the 181 patients with CAP and PPE, we found that 95 patients had measurements of $<2.5 \mathrm{~cm}$ on CCT. The outcomes of these 95 patients with effusions measuring $<2.5 \mathrm{~cm}$ are summarised in table 2 . There were 31 negative outcomes occurring in $29.5 \%$ (28 out of 95) of CAP patients with PPE, with all but five of these poor outcomes were attributable to causes other than PPE.

Of the five patients with negative outcomes not easily attributable to other causes, outcome was death in four patients admitted to a hospice and who had discontinuation of antibiotics or inadequate documentation. The three remaining poor outcomes were due to interventions, possibly implying a complicated effusion or empyema (fig. 3). In the three outcomes with pleural thoracentesis indicating complicated effusion or empyema, tube thoracostomy, thorocotomy or decortication, two cases were not attributable to the effusion. The first patient had a central line inadvertently placed into the pleural space with infusion of normal saline that required urgent thoracotomy. The second patient had thoracentesis suggesting possible PPE but also had adenocarcinoma invading the pleural space and chest wall with associated post-obstructive pneumonia, the effusion was found to be malignant and not related to the pneumonia. The patient

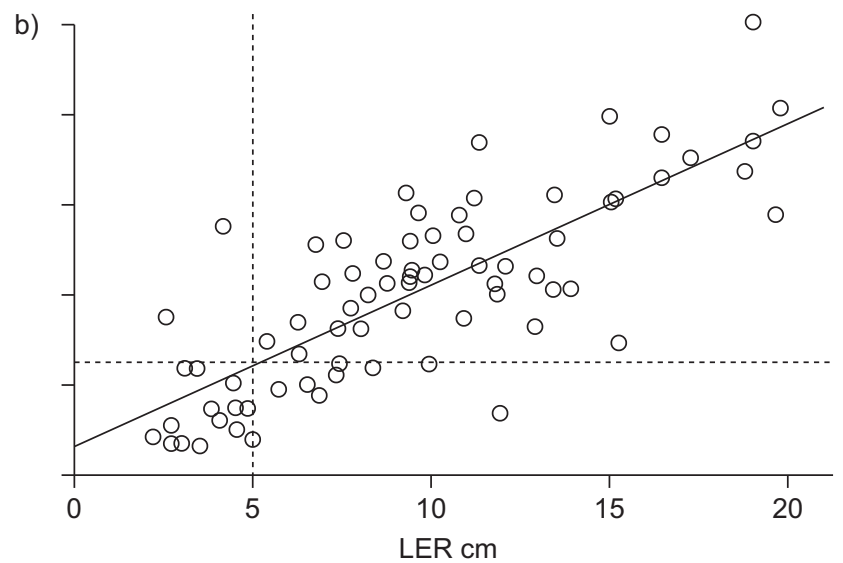

FIGURE 3. Correlation of pleural effusion measurements between a) 79 patients with chest computed tomography (CCT) and lateral decubitus radiograph (LDR) (linear $\left.r^{2}=0.626\right)$ and b) 76 patients with CCT and lateral erect radiograph (LER) (linear $r^{2}=0.623$ ). Horizontal dotted line $=2.5 \mathrm{~cm}$. 


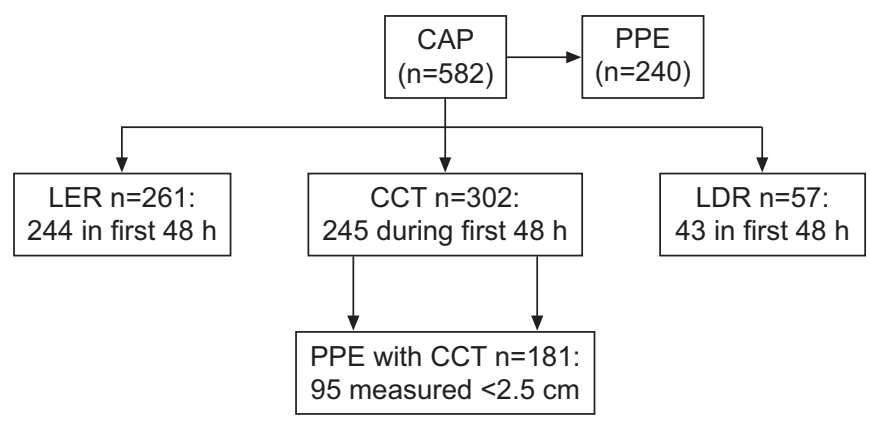

FIGURE 4. The distribution of radiographs in patients with communityacquired pneumonia (CAP) with regard to timing and number of patients evaluated. PPE: parapneumonic effusion; LER: lateral erect radiograph; CCT: chest computed tomography; LDR: lateral decubitus.

received no drainage procedure and suffered no ill outcomes. The remaining patient had a complicated PPE with a pleural fluid $\mathrm{pH}$ of $6.89, \mathrm{LDH}$ of $7,914 \mathrm{mg} \cdot \mathrm{dL}^{-1}$, total protein $4.90 \mathrm{mg} \cdot \mathrm{dL}^{-1}$ and glucose $21 \mathrm{mg} \cdot \mathrm{dL}^{-1}$, and received chest tube drainage, which was reported to drain purulent material. The LDR measurement in this patient was $0.93 \mathrm{~cm}$, while the CCT measurement was $2.45 \mathrm{~cm}$.

In patients with CAP and PPE, 43 underwent both LDR and CCT. LDR was performed after initial CCT in $27(62.8 \%)$ of these patients. Loculations were found in $24(10 \%)$ out of 240 CAP patients with PPE, all of whom were detected by CCT during their initial evaluation. Of these 24 patients, eight underwent both LDR and CCT. Of these eight patients, only five had partial loculations with persistent layering that prevented detection by LDR but still had measurements indicating the need for thoracentesis. One of these missed loculated effusions was $<1 \mathrm{~cm}$ in size and would not have been tapped without CCT. Its aspiration was consistent with complicated PPE or empyema. LDR detected only one of nine cases of loculated effusion as determined by CCT. In all of these cases, LDR measured $>5 \mathrm{~cm}$ and aspiration was indicated regardless of the presence of loculation.

\section{DISCUSSION}

By correlating measurements between different imaging modalities in patients with PPE, we discovered that a measurement of $2.5 \mathrm{~cm}$ by CCT was equivalent to established measurements by
LDR and LER. By applying our new measurement to only those patients hospitalised with CAP and PPE, we found that a PPE measuring $<2.5 \mathrm{~cm}$ on CCT could be managed conservatively without thoracentesis. We also found that in our hospitalised CAP patients, CCT was the initial imaging modality in $\sim 40 \%$ of cases. When PPE was discovered by CCT, $>60 \%$ of these patients received subsequent imaging with LDR. When CCT is available during the first $24 \mathrm{~h}$ of hospitalisation, it is probably more efficient to use a measurement of $2.5 \mathrm{~cm}$ to determine the need for thoracentesis than to delay intervention to wait for LDR, which may be associated with increased morbidity and mortality, and should be avoided $[3,4,10,11]$.

The utility of estimating relevant parapneumonic pleural volumes through radiographic measurements was established by LigHT et al. [2]. Similar to that seminal paper, we found that negative outcomes were common in patients with effusions, but that effusion progression was rare when effusions were small (CCT measurements $<2.5 \mathrm{~cm}$ ). Unlike the original LDR study, we found a single case of disease progression. However, this failure occurred despite both a measurement of $<2.5 \mathrm{~cm}$ on CCT and $<1 \mathrm{~cm}$ on LDR. Given the close approximation of this case's measurement to both values, we feel this represents an unusual occurrence and underscores the importance of clinical judgment and repeat evaluation in borderline cases, regardless of the means of measurement.

METERSKY [8] provided evidence that effusions $>5 \mathrm{~cm}$ in height on an LER require thoracentesis; this also correlated with our CCT value of $2.5 \mathrm{~cm}$. Like METERSKY [8], we feel that LDR may lead to delays in patient care when other modalities, such as LER or CCT, are readily available $[8,10]$. In fact, our study found that the majority of patients received a CCT prior to LDR. Our study benefited from a larger population of patients compared with the study by METERSKY [8] and, thus, provides a more reliable linear regression model. As our LDR and LER values provided similar CCT measurements, it supports the previous assertion that LER measurements of $5 \mathrm{~cm}$ are an alternative to LDR. Compared with LER, CCT had additional benefits, including a successful interpretation rate of $95 \%$ (table 1), and allowed better visualisation of loculations and septations. In our CAP patients, LER failed to demonstrate $88.9 \%$ of loculations visualised by CCT. However, due to the size of their effusions, all of these patients received

TABLE 1 Available radiographs for comparison in community-acquired pneumonia patients with parapneumonic effusion and their limitations

\begin{tabular}{|c|c|c|c|}
\hline & LDR & LER & ССТ \\
\hline Total radiographs & 57 & 90 & 181 \\
\hline Measurable radiographs & $50(87.7)$ & $64(71.1)$ & $148(81.8)$ \\
\hline Loculation demonstrated & 3 & 1 & 24 \\
\hline Uninterpretable & $4(7.0)$ & $25(28.8)$ & $9(5.0)$ \\
\hline Limitation of interpretation & $\begin{array}{l}\text { Incorrect side } n=2 \text {, } \\
\text { incorrect radiograph type } n=1 \text {, } \\
\text { massive effusion } n=1\end{array}$ & $\begin{array}{l}\text { Bilateral effusion } n=13 \text {, } \\
\text { inadequate visualisation of sulcus } n=6 \text {, } \\
\text { under penetrated } n=4 \text {, } \\
\text { post-thoracentesis } n=1 \text {, } \\
\text { diaphragm elevation } n=1\end{array}$ & $\begin{array}{l}\text { Post-thoracentesis } n=4 \text {, } \\
\text { contralateral effusion } n=2 \text {, } \\
\text { hydropneumothorax } n=1 \text {, } \\
\text { massive effusion } n=1 \text {, } \\
\text { image missed } n=1\end{array}$ \\
\hline
\end{tabular}

Data are presented as $\mathrm{n}$ or $\mathrm{n}(\%)$. LDR: lateral decubitus radiograph; LER: lateral erect radiograph; CCT: chest computed tomography. 


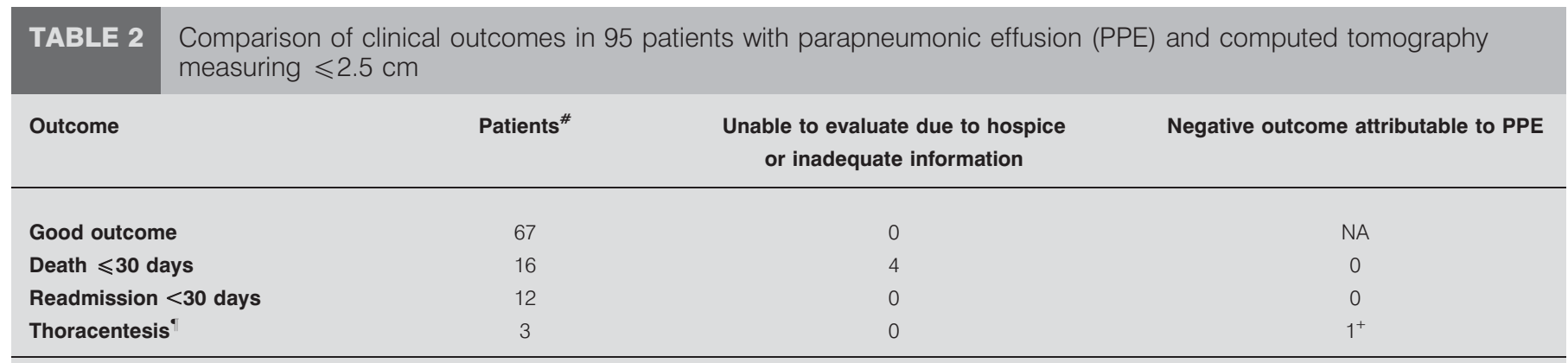

Data are presented as n. NA: not applicable. "*: three patients had two outcomes; ' : indicating complicated effusion or empyema, tube thoracostomy, thoracotomy or decortication; ${ }^{+}$: measured $2.45 \mathrm{~cm}$ by chest computed tomography and $0.93 \mathrm{~cm}$ by lateral decubitus radiograph.

thoracentesis, so this had little clinical significance. In contrast to LER, we report a case of LDR measurements that neither indicated the need for thoracentesis nor detected loculations but, in fact, had a complicated PPE.

While several prior studies have attempted to use either CCT or magnetic resonance imaging to evaluate pleural effusions, these techniques have only proven efficacious at differentiating transudative from exudative effusions, but cannot distinguish infected from uninfected exudative effusions [16-19]. While ultrasound can assess fluid volume in a similar way to LDR and is advantageous because it can be performed on medically unstable patients and can guide the placement of tubes into loculated effusions [20], it is limited by its inaccuracy when assessing small-volume effusions [10] and its failure to recognise purulent collections in a significant portion of patients [18, 21]. Therefore, measurement by either LDR or LER and subsequent aspiration is still required in the assessment of PPE; measurements by CCT provide an additional means of evaluating clinically significant effusions and in aiding clinical judgment.

It is important to note that our study has several potential limitations. Notably, our study was not prospective in nature and some outcomes could not be assessed due to enrolment in a hospice or lack of adequate documentation. When measuring radiographic images, reviewers were not blinded to the measurements of the other radiographs. While this could introduce some bias into our results we feel this risk is minimised by the application of our generated measurement to clinical outcomes. Our results should also be applied to patients with HAP and VAP with caution, as our outcome measurements were only possible in those with CAP. The primary strengths of our study lie in the number of patients used to generate our linear regression models and the near identical values for CCT produced by both LDR and LER regression. Furthermore, our study provides an assessment of clinical outcomes when applying this novel measurement. While our study was limited to an elderly population that was almost exclusively male, we feel that their significant comorbidities characterise most patients hospitalised with CAP by evaluation of the Pneumonia Severity Index score [22]. It is possible that, due to differences in pleural space volume between males and females, our CCT measurement of $2.5 \mathrm{~cm}$ may overestimate the size of an effusion in female patients.

Recently, due to the superiority of this technique, there has been an increase in the usage of CCT in the evaluation of hypoxic patients to exclude pulmonary embolism [23]. Although we feel that LDR still has an important role in the evaluation of PPE, it can lead to a delay in care when CCT is already available. It is our belief that patients with CCT measurements of $>2.5 \mathrm{~cm}$ should receive thoracentesis without additional imaging. We do caution interpretation of any measurements that fall very near cut-off values, and recommend repeat clinical evaluation and imaging. This study would be best substantiated by a prospectively designed study to see if it does in fact reduce cost and expedite time to thoracentesis.

In conclusion, when CAP patients have a CCT readily available, measurements of $<2.5 \mathrm{~cm}$ can be conservatively managed without thoracentesis. We feel it is pertinent to conclude with an alteration to the famous saying of SAHN and LiGHT [24]: "The sun should never set on a parapneumonic effusion", unless that effusion measures $<2.5 \mathrm{~cm}$ on CCT.

\section{STATEMENT OF INTEREST}

None declared.

\section{ACKNOWLEDGEMENTS}

This study was performed with the assistance of the Robley Rex Louisville Veteran Affairs Hospital (Louisville, KY, USA).

\section{REFERENCES}

1 Fine NL, Smith LR, Sheedy PF. Frequency of pleural effusions in mycoplasma and viral pneumonias. N Engl J Med 1970; 283: 790-793.

2 Light RW, Girard WM, Jenkinson SG, et al. Parapneumonic effusions. Am J Med 1980; 69: 507-512.

3 Bartlett JG, Finegold SM. Anaerobic infections of the lung and pleural space. Am Rev Respir Dis 1974; 110: 56-77.

4 Huang HC, Chang HY, Chen CW, et al. Predicting factors for outcome of tube thoracostomy in complicated parapneumonic effusion or empyema. Chest 1999; 115: 751-756.

5 Light RW, Macgregor MI, Luchsinger PC, et al. Pleural effusions: the diagnostic separation of transudates and exudates. Ann Intern Med 1972; 77: 507-513.

6 Brewin A, Arango L, Hadley WK, et al. High-dose penicillin therapy and pneumococcal pneumonia. JAMA 1974; 230: 409-413.

7 Light RW. Management of parapneumonic effusions. Arch Intern Med 1981; 141: 1339-1341.

8 Metersky ML. Is the lateral decubitus radiograph necessary for the management of a parapneumonic pleural effusion? Chest 2003; 124: $1129-1132$.

9 Brenner DJ, Hall EJ. Computed tomography: an increasing source of radiation exposure. $N$ Engl J Med 2007; 357: 2277-2284. 
10 Heffner JE, McDonald J, Babieri C, et al. Management of parapneumonic effusions. Arch Surg 1995; 130: 433-438.

11 Landay MJ, Christensen EE, Bynum LJ, et al. Anaerobic pleural and pulmonary infections. AJR Am J Roentgenol 1980; 134: 233-240.

12 Eibenberger KL, Dock WI, Ammann ME, et al. Quantification of pleural effusions: sonography versus radiography. Radiology 1994; 191: 681-684.

13 Ramirez J, Aliberti S, Mirsaeidi M, et al. Acute myocardial infarction in hospitalized patients with community-acquired pneumonia. Clin Infect Dis 2008; 47: 182-187.

14 Colice GL, Curtis A, Deslauriers J, et al. Medical and surgical treatment of parapneumonic effusions: an evidence based guideline. Chest 2000; 118: 1158-1171.

15 Niederman MS, Mandell LA, Anzueto A, et al. Guidelines for the management of adults with community-acquired pneumonia. Am J Respir Crit Care Med 2001; 163: 1730-1754.

16 Davis SD, Henschke CI, Yankelevitz DF, et al. MR imaging of pleural effusions. J Comput Assist Tomogr 1990; 14: 192-198.

17 Baysal $\mathrm{T}$, Bolut $\mathrm{T}$, Gokirmak $\mathrm{M}$, et al. Diffusion weighted MR imaging of pleural fluid: differentiation of transudative vs exudative effusions. Eur Radiol 2004; 14: 890-896.
18 Kearney SE, Davies CW, Davies RJ, et al. Computed tomography and ultrasound in parapneumonic effusions and empyema. Clin Radiol 2000; 55: 542-547.

19 Maffessanti M, Tommasi M, Pelligrini P. Computed tomography of free pleural effusions. Eur J Radiol 1987; 7: 87-90.

20 Raptopoulos V, Davis LM, Lee G, et al. Factors affecting the development of pneumothorax associated with thoracentesis. AJR Am J Roentgenol 1991; 156: 917-920.

21 Kurian J, Levin TL, Han BK, et al. Comparison of ultrasound and $\mathrm{CT}$ in the evaluation of pneumonia complicated by parapneumonic effusion in children. AJR Am J Roentgenol 2009; 193: 1648-1654.

22 Fine MJ, Auble TE, Yealy DM, et al. A prediction rule to identify low-risk patients with community acquired pneumonia. $N$ Engl J Med 1997; 336: 243-250.

23 Stein PD, Woodard PK, Weg JG. Diagnostic pathways in acute pulmonary embolism: recommendations of the PIOPED II investigators. Radiology 2007; 242: 15-21.

24 Sahn SA, Light RW. The sun should never set on a parapneumonic effusion. Chest 1989; 95: 945-947. 